\title{
GCU
}

Glasgow Caledonian

University

University for the Common Good

\section{Toward a cloud computing learning community}

Foster, Derek ; White, Laurie; Erdil, Cenk; Adams, Joshua; Nazir, Sajid; Argüelles, Amadeo; Hainey, Brian; Hyman, Harvey; Lewis, Gareth; Nazir, Sajid; Van Nguyen; Sakr, Majd; Stott, Lee

Published in:

ITiCSE-WGR '19: Proceedings of the Working Group Reports on Innovation and Technology in Computer Science Education

DOI:

$10.1145 / 3344429.3372506$

Publication date:

2019

Document Version

Author accepted manuscript

Link to publication in ResearchOnline

Citation for published version (Harvard):

Foster, D, White, L, Erdil, C, Adams, J, Nazir, S, Argüelles, A, Hainey, B, Hyman, H, Lewis, G, Nazir, S, Van Nguyen, Sakr, M' \& Stott, L 2019, Toward a cloud computing learning community. in ITiCSE-WGR '19:

Proceedings of the Working Group Reports on Innovation and Technology in Computer Science Education. Association for Computing Machinery (ACM), pp. 143-155, Innovation and Technology in Computer Science Education 2019, Aberdeen, United Kingdom, 15/07/19. https://doi.org/10.1145/3344429.3372506

\section{General rights}

Copyright and moral rights for the publications made accessible in the public portal are retained by the authors and/or other copyright owners and it is a condition of accessing publications that users recognise and abide by the legal requirements associated with these rights.

Take down policy

If you believe that this document breaches copyright please view our takedown policy at https://edshare.gcu.ac.uk/id/eprint/5179 for details of how to contact us. 


\section{Toward a Cloud Computing Learning Community}

\author{
Derek Foster \\ School of Computer Science \\ University of Lincoln \\ Lincoln, UK \\ defoster@lincoln.ac.uk \\ Joshua Adams
Donald R. Tapia College of Business
CS Department
Saint Leo University
Saint Leo, FL, USA
joshua.adams03@saintleo.edu
Harvey Hyman
MUMA College of Business
University of South Florida
Tampa, FL, USA
hhyman@usf.edu

$$
\text { Van Nguyen }
$$
Donald R. Tapia College of Business
CS Department
Saint Leo University
Saint Leo, FL, USA
van.nguyen@saintleo.edu

\author{
Laurie White* \\ Google LLC \\ Seattle, WA, USA \\ lauriewhite@google.com
}

\author{
Amadeo Argüelles \\ Centro de Investigación en \\ Computacion \\ Instituto Politècnico Nacional \\ Mexico City, Mexico \\ jamadeo@cic.ipn.mx

\section{Gareth Lewis} \\ Falmouth University \\ Falmouth, Cornwall, UK \\ gareth.lewis@falmouth.ac.uk
}

Majd Sakr

School of Computer Science

Computer Science Department

Carnegie Mellon University

Pittsburgh, PA, USA

msakr@cs.cmu.edu

\author{
D. Cenk Erdil ${ }^{*}$ \\ School of Computer Science \& \\ Engineering \\ Sacred Heart University \\ Fairfield, CT, USA \\ erdild@sacredheart.edu \\ Brian Hainey \\ Glasgow Caledonian University \\ Glasgow, Scotland, UK \\ b.hainey@gcu.ac.uk
}

\author{
Sajid Nazir \\ Glasgow Caledonian University \\ Glasgow, Scotland, UK \\ sajid.nazir@gcu.ac.uk
}

\author{
Lee Stott \\ Microsoft \\ Seattle, WA, USA \\ leestott@microsoft.com
}

\begin{abstract}
Cloud computing continues to be an important technology in higher education. This domain is a rapidly evolving space, and continues to gain momentum as a primary infrastructure topology for technological advances across emergent industries. The on-thecloud paradigm provides numerous affordances and new methods of working in industry, and also for end users within recent fields of study, such as machine learning and artificial intelligence. As we move into the Industry 4.0 era with technology pillars such as the internet of things and cybersecurity, the importance of skillsets for cloud-based services will be an essential attribute for the majority of technology-related professions. Many higher education institutions have focused on offering training opportunities and programs for cloud computing, however, a lack of high-quality, contextualized to industry, curricula materials continues to be a challenge for educators. The purpose of this paper is to report on analyses conducted to categorize cloud computing courses currently taught in the higher education sector and to determine the possibility of moving towards the goal of a model curriculum. Additionally, the paper aims to provide guidance to educators about cloud computing skillsets sought in the job market, and to report on a community platform designed to host cloud learning resources.
\end{abstract}

\section{CCS CONCEPTS}

- Applied computing $\rightarrow$ Education; • Computer systems organization $\rightarrow$ Cloud computing;

\section{KEYWORDS}

Cloud computing, education, computer science, curriculum development

\section{ACM Reference Format:}

Derek Foster, Laurie White, D. Cenk Erdil, Joshua Adams, Amadeo Argüelles, Brian Hainey, Harvey Hyman, Gareth Lewis, Sajid Nazir, Van Nguyen, Majd Sakr, and Lee Stott. 2020. Toward a Cloud Computing Learning Community. In Proceedings of ACM Conference (Conference'17). ACM, New York, NY, USA, 13 pages. https://doi.org/10.1145/nnnnnnn.nnnnnnn 


\section{INTRODUCTION}

Cloud technologies are constantly evolving to support industry and business innovation, and are instrumental in supporting major technology areas such as the internet of things, artificial intelligence and machine learning, cybersecurity, and big data [33]. Despite significant growth and uptake in cloud services by industry, the education sector has lagged behind in providing industry-relevant cloud courses resulting in a cloud skills shortage [38]. This cloud course deficit was identified in the 2018 ITiCSE Cloud Working Group (WG) report [13], where the major accomplishment of the group was the development of Knowledge Areas (KAs) and Learning Objectives (LOs) for each KA (See Appendix A for the list of KAs). The produced KAs and LOs could be adopted by educators to support the design and uptake of cloud computing courses.

This paper reports on the work of a 2019 ITiCSE WG to expand on the work of the 2018 WG with the aim of creating a communitydriven repository containing metadata on high-quality cloud learning materials and courses. Such a repository would enable educators to search and apply filters to find the most suitable in-depth cloud course material to include in their curricula. A primary benefit of such a repository would be alleviating the significant difficulties and challenges around creating learning materials for such a fastmoving technology space. Additionally, the WG understands the need to support users of the repository and proposes a community to provide support and guidance.

In order to arrive at the design and deployment of a suitable repository and community for cloud learning materials, the WG carried out three main tasks, with findings of each presented in this paper:

- Existing course analysis to analyse existing cloud curricula to determine particular course categories and the concentrations of specific cloud-related topics;

- Job market analysis to perform a career-driven analysis to identify gaps between cloud-related training and industry needs, and provide a mapping of how they relate to each other;

- Learning community creation to design a learning resources repository built with a supporting community, to help other individuals to join, contribute, ask and answer questions, and share the knowledge they have gained.

The findings of the existing course analysis and job market analysis tasks feed directly into the design of the repository proposed by the learning community creation task.

\section{BACKGROUND}

Higher education institutions delivering computer science programs commonly use online learning environments to host and deliver a range of learning resources to support students with their studies [5, 19, 25, 27, 32]. Generally, these are gated silos of information with access limited to staff and students. The learning community in this sense is at the macro level, and contained within the institution's departments and colleges, with the resources not readily made publicly available. This approach does not support a cross-institution learning commons ethos that encourages scholarly collaboration with other institutions to publicly share learning approaches and materials [26]. Effectively, institutions adopt a closed stance with valuable knowledge sealed off from external community contribution, uptake, and sharing. Additionally, even when faculty have materials on their school WWW sites, they may not have it licensed to permit reuse [18]. This inhibits the widespread uptake of teaching currently in-demand technology areas, in this case cloud computing, that require significant expertise to develop and adopt [10,35]. Additionally, there may be institutional policies that limit, or even prohibit, the sharing of learning materials created by academics whilst at that institution. As a result, there is likely a corpus of high quality learning materials behind gated silos that could have a significant positive impact if shared widely with other educators. Academic work on an incremental evolution of the learning commons, termed disciplinary commons [39], promotes the concept of community-driven repositories of teaching materials that facilitates collaboration and sharing of resources.

The development of new learning materials for complex and emergent areas of computer science is a significant barrier for educators to rapidly adopt and deliver in their home institution. When learning about cloud computing, theoretical knowledge of the discipline topics such as virtualisation, load balancing, and storage optimisation is important, but perhaps more importantly it requires practical competencies. This does not mean it is not possible to run cloud courses that are mainly theory-based, as there is a significant body of academic concepts to utilise in their development. It would be helpful for educators to be able to distinguish between primarily theory-based and practical-based cloud courses when searching for learning materials to better fit into their overall curricula. To cater to a wider educator audience, it makes sense for a repository of cloud learning materials to detail a range of different courses, discoverable through tags to identify their suitability as purely academic courses, practice-based, or a combination. For example in the UK, research-intensive institutions that are focused on producing world-class research such as those in the Russell Group may be more aligned to courses with significant academic content [31], with institutions in the University Alliance group more aligned to learning resources that support their focus providing a technical and professional education [43].

\section{RELATED WORK}

For the purposes of designing a community-driven repository, it is expected that educators would serve as both consumers and contributors of content, with the target audience primarily computer science (CS) educators. A consumer essentially searches for and consumes resources from the repository, while a contributor will produce resources for the repository. This section will discuss related academic work around the design and development of online communities for sharing teaching resources, as well as examples of currently deployed repositories used in CS.

\subsection{Disciplinary Commons}

Academic literature around online communities for educators to collaborate and share learning resources has been well developed in the last 10 years. Early work by Tzikopoulos et al. [41] coined the term Learning Object Repositories (LORs), as spaces to share relevant pedagogical materials to support teaching and learning. The design of such tools to support communities are not without 
their problems, in terms of ease-of-use and sustaining engagement and uptake. More recently, the idea of a disciplinary commons [39] has been explored with the following aims:

- To document and share knowledge about teaching and student learning;

- To establish practices for the scholarship of teaching by making it public, peer-reviewed, and amenable for future use and development by other educators.

Further work by Ni et al. [28] identified desirable attributes that CS educators wanted as a member of the community to be:

- Belonging to a community of CS teachers;

- Feeling confident and affirmed in CS teaching;

- Seeing the value of sustained community with other CS teachers.

Such important community attributes will influence the repository the target audience will engage with, in order to create a positive community experience. Similar to the findings by $\mathrm{Ni}$ et al., work by Ray et al. [30] also highlighted the importance of educators' confidence when deciding to make a community contribution. In a general sense, engagement theory suggests that two of the major conditions commonly required for community members to feel engaged are personal/group values and support from others [23]. In summary, by informing the design of the proposed repository to support the educator attributes of belonging, confidence and revalidation, and the value of a sustained community, the repository should avoid the pitfalls of disengagement and lack of community buy-in.

\subsection{CS Repositories}

From the educator's perspective, teaching materials for CS courses can be time-consuming and difficult to develop, particularly for emergent areas where a public knowledge-base may not be available. An online, community-driven repository would facilitate sharing of curated material that educators can use on a self-service basis. Some examples of successful teaching repositories are: CSinParallel [6], TCPP Curriculum Initiative on Parallel and Distributed Computing [29], and dbdc: Creating a Disciplinary Commons in Computing Education [8].

While the listed teaching repositories have seen suitable engagement levels, there are practicalities to be considered for their management and long-term sustainability. More specifically, as custom-built repositories they have hosting and management requirements, thereby creating an administrative burden. From the outset, one of the aims of the WG was to use a commonly available platform to host the repository in order to reduce development, management, and maintenance overheads.

Work by Fincher et al. [12] investigated the potential causes of success and failure for teaching repositories and reported on the importance of curation, content, contribution, community, catalogue, and control as enablers for a successful and engaged teaching repository community. Whilst the previously mentioned teaching repositories address some of the community and engagement attributes outlined by $\mathrm{Ni}$ et al., and Fincher et al., there are several key attributes missing from them. There is no standardised realtime communication (for communication and file sharing) through chat/group clients or optimised search functions. The WG members believe the addition of rich communication tools for groups and search functions with tagging can greatly enhance the design of CS repositories where community is a primary focus. This work acknowledges the importance of the discussed literature, in order to design and deploy a successful repository for cloud learning resources. In addition, work carried out for each of the WG objectives also informs the design and interactive attributes of the repository.

\section{METHODS}

In the months leading up to the face-to-face meeting at ITiCSE, members of the WG took on the following primary tasks:

- Analyze existing cloud courses;

- Analyze the cloud computing job market;

- Create a learning community..

The methods to achieve completion of each individual task are discussed here.

\subsection{Analyzing Existing Cloud Courses}

The objectives for the existing cloud course analysis subteam were to:

- Create a standardized approach on global differences when identifying academic course/module terminology to avoid conflation;

- Gather information on existing cloud courses previously or currently being delivered at educational institutions globally;

- Analyze the course data gathered to determine if courses can be categorized by themes in a meaningful way;

- Report outcomes of the analysis including detailed descriptions of each identified course theme;

- Consider emerging themes and identify gaps compared to those identified.

The subteam performed a landscape analysis of the current state of cloud computing courses by conducting a survey of faculty who self identify as teaching or creating cloud learning materials. Additionally, a manual trawl of online educational course portals, such as UCAS [42], provided extra course information to supplement the survey data.

4.1.1 Standardizing Existing Cloud Course Terminology. Throughout the world, university students are typically enroled on creditbearing blocks of teaching while studying to gain a bachelor's or master's degree. Each block of teaching, if completed successfully, provides the student with a specific amount of credit which counts towards the total required for the award of their degree. The terminology used for blocks of teaching varies globally, with the most common terms in use being course and module. The term course is used in a range of countries including the US and Canada and the term module is used in a range of countries including the UK and Australia. A course/module has a fixed duration, may be elective or compulsory and has a variable amount of credit associated with it. In some countries where the term module is used, the collection of modules leading towards a degree can be called a course. Elsewhere, the collection of courses/modules is called a program/programme. The analysis undertaken here, for simplicity, uses course and program throughout, where course is a component of an overall program of study. In addition to deciding on the term program to form 
an overall collection of courses, a decision was made to use the term graduate rather than postgraduate for programs leading to an master's award and undergraduate for programs leading to a bachelors degree. A decision on this was required since the term postgraduate is also used in many parts of the world.

4.1.2 Existing Course Survey. A survey was designed to be administered to faculty who currently teach cloud courses. The data gathered for the survey will not be used outside the scope of this work. Members of the WG were available during the period when the survey was live to answer questions as they arose. The nature of the data gathered did not require ethical approval.

The survey questions included: the name of the institution, instructors, course overview, description, syllabus, learning outcomes, link to the course, course name and ID, assignment details, and any teaching resources they would be willing to share.

The survey focused on gathering information on the following curriculum topics:

- The use of hands-on projects and the percentage of the grade associated with this. (The caveat is that hands-on training is considered to be an effective learning technique, while it's more expensive and difficult to create and assess);

- The employment of public/commercial cloud vendor offerings and which specific services are utilized;

- The delivery methods used such as face-to-face, hybrid and online (since this can affect the type of assignments and assessments that are administered);

- The types and weightings of all course assessments;

- The specific cloud technical topics and curriculum covered such as cloud cybersecurity, introductory cloud topics, cloud development, DevOps and software engineering cloud topics.

The survey was designed to account for a global audience of faculty with diverse learning contexts.

A pilot of the survey with WG members was carried out to uncover ambiguities and to ensure the capture of relevant information for different types of cloud curriculum courses. This resulted in additional questions to uncover aspects of delivery that were not included in the pilot version of the survey. Various approaches were used to ensure widespread dissemination of the survey to ensure the varying perspectives prevalent in cloud course development were captured. Initially, specific university faculty known to be involved in teaching cloud computing courses were contacted through WG members. The survey was later advertised using SIGCSE and ITiCSE distribution lists for faculty involved in teaching cloud computing. A copy of the survey is in Appendix B.

4.1.3 Manual Search for Course Information. It was expected that responses to the survey might not provide all the required course in formation and a manual search to gather more information was also performed using keywords such as "cloud, curriculum, virtualization", and specific university names, to further support identifying relevant course information.

\subsection{Analyzing the Cloud Job Market}

For this task, a landscape analysis of the cloud computing job market was conducted, using data from publicly advertised job listings containing cloud computing in the advertisement title and/or description. The aim of the task was twofold: i) identify the major cloud job role types, and group identified sub-roles within them, and ii) map the derived major job roles to the KAs produced from the previous working group [13], as well as the course characterizations produced from the existing cloud course analysis task.

4.2.1 Identifying Cloud Job Roles and Sub-roles. To identify major cloud job roles, data was searched and collected from posts advertised on popular, large-scale recruitment services such as Glassdoor, CWJobs, Dice, LinkedIn, Indeed, and CareerBuilder [7, 9, 17, 21, 24, 40]. Keywords used were limited to cloud, and cloud computing. To group cloud sub-roles to major roles, pre-populated job titles on the Glassdoor and CWJobs recruitment sites were used as the search keywords, for example, job titles such as Cloud Architect, DevOps Engineer, and Cloud Orchestration Architect.

4.2.2 Mapping Job Roles to KAs and Course Themes. Mapping major cloud job roles to KAs and the course themes developed by the existing course analysis subteam is intended to assist current and future students in understanding the importance of cloud-related skills in industry such as cybersecurity, machine learning, and artificial intelligence.

\subsection{Creating a Learning Community Repository}

The community repository was not designed in isolation and was created from the outcomes of a literature survey and findings from the existing cloud courses and cloud computing job market analysis tasks.

A literature survey was carried out to understand best practices when designing learning resource repositories, with findings informing the key educator interactions with the proposed repository for searching, making a contribution, and communication. This approach is to ensure the best possible chance of success in uptake and continuous community engagement.

Additionally, suitable hosting and communication platforms were investigated based on the following goals:

- Low (preferably zero) cost;

- Easy access for consumers including the ability to search and filter;

- Rich communication features for groups;

- The ability to review submissions before they are added to the repository;

- Easy access for contributors;

- Integration between repository and communication tool;

- The ability to see a history of changes;

- The ability to see the history of uses;

- The ability of users to provide feedback on submissions.

Such goals when achieved would facilitate the desired educator and community interactions throughout the repository.

A contribution template was produced that captures appropriate course metadata for uploading to the repository. This template has been tested by WG members to ensure relevant data is captured with appropriate filters and custom tagging. 


\section{RESULTS}

This section will discuss the gathered data for the existing cloud course analysis and cloud computing job market analysis, followed by the findings for understanding the design and deployment of a learning community. The purpose of this approach enables the results of the major tasks to inform the goal of creating a communitydriven repository.

\subsection{Existing Course Analysis}

A factor to be considered when looking at courses on a global basis is the determination of the level of study. This required consultation of the different standards and frameworks used worldwide. The global diversity of the 12 WG members helped to contextualise this geographically, however, it was necessary to consult standards documents such as the European Qualifications Framework (EQF) [11], the Scottish Credit and Qualification Framework (SCQF) [34], and the Regulated Qualification Framework (RQF) [4] to provide a broader view of the various level of study. This was specifically done to help frame what constitutes a higher education level of study.

5.1.1 Manual Search of Cloud Curricula. To compliment the survey data set, an online, manual search of cloud computing courses was carried out. Although many of the courses found through the manual search had "virtualization" or "cloud" in their title, only a subset of courses had a major focus on specific cloud topics and could be included in the data. Some courses found were introductory, covering a broad range of topics, while others were specialty computer science courses that included only a minimum selection of cloud topics. This indicates faculty are progressing in their efforts to include cloud topics in their existing courses, likely to satisfy the growing demand from the student body and industry for cloudrelated skills. This data supports the WG in providing a repository containing appropriate reference content.

5.1.2 Data Analysis. In response to the survey, 33 submissions were received. Additionally, 30 courses were collected through a manual search. A combined total of 63 courses were collected with 7 discarded due to incompleteness. The total number of courses used, both survey responses and manual search during the analysis, was 56 .

The course survey responses varied from those providing a full course description, curriculum details and supporting documentation, to those providing very general course descriptions with little detail available to determine whether the course contained cloud topics that could be used for categorization. In cases where detail was missing, the response was discarded. In some cases, the submission covered a complete undergraduate or graduate program rather than being a component course in a program. These too were discarded. The survey responses with relevant cloud content were used as a starting point for categorization through an analysis of topic areas covered and the overall focus of the course. Each course was discussed in detail by WG members and the course information reviewed in order to accurately determine if categorization was possible. Careful consideration of the course description, learning outcomes, syllabus, assessments, and web page content when available was carried out to reach a consensus on the overall single

theme of the course as a potential for categorization. In general, it was found that the majority of courses had a dominant area of focus that is covered in-depth, accompanied by one or more peripheral supporting areas identified in the 2018 WG report Knowledge Areas (KAs) [13]. The decision to categorize courses based on a single dominant theme rather than a main and multiple supporting themes was agreed as the best approach by the WG members. Such an approach would facilitate optimal searching of, and contribution of materials on the proposed repository.

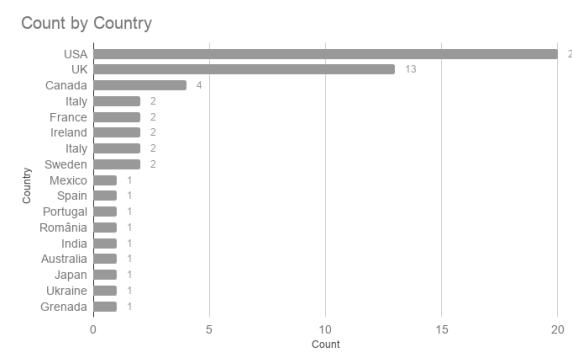

Figure 1: Survey and manual search results by country
Figure 1 shows the course distribution globally. Some geographical areas are not well covered due to not receiving survey entries or the manual search not yielding results of related courses in these locations. However, the purpose of this work was not necessarily to obtain global course coverage, rather to obtain a representative sample from as wide a range of countries as possible so categorization would be representative within the search parameters. It is anticipated that coverage will improve as the repository is adopted and populated by educators from other countries.

Table 1: Survey and search results by country and program level

\begin{tabular}{|c|c|c|c|c|c|}
\hline $\begin{array}{l}\text { Combined } \\
\text { Survey } \\
\text { and Search } \\
\text { Country }\end{array}$ & $\begin{array}{l}\text { Graduate } \\
\text { Only }\end{array}$ & $\begin{array}{l}\text { Professional } \\
\text { Only }\end{array}$ & $\begin{array}{l}\text { Undergraduat, } \\
\text { Only }\end{array}$ & $\begin{array}{l}\text { Undergraduat } \\
\text { Graduate }\end{array}$ & Grand Total \\
\hline Australia & & & 1 & & 1 \\
\hline Canada & 4 & & & & 4 \\
\hline France & 2 & & & & 2 \\
\hline Germany & & & 1 & & 1 \\
\hline Grenada & & & 1 & & 1 \\
\hline India & & & 1 & & 1 \\
\hline Ireland & 2 & & & & 2 \\
\hline Italy & 1 & & 1 & & 2 \\
\hline Japan & 1 & & & & 1 \\
\hline Lithuania & & & 1 & & 1 \\
\hline Mexico & & & & 1 & 1 \\
\hline Portugal & & & 1 & & 1 \\
\hline Romania & 1 & & & & 1 \\
\hline Spain & & & & 1 & 1 \\
\hline Sweden & 1 & & 1 & & 2 \\
\hline UK & 5 & & 7 & 1 & 13 \\
\hline Ukraine & 1 & & & & 1 \\
\hline USA & 9 & 1 & 5 & 5 & 20 \\
\hline Grand Total & 27 & 1 & 20 & 8 & 56 \\
\hline
\end{tabular}

Table 1 illustrates the course levels of study analyzed from a coarsegrained perspective where the only classifications used are undergraduate (bachelor's), graduate (master's), and professional. An alternative approach aligned with one of the standards covered at the start of this section is possible, however, this was not considered necessary for those who would use the repository. Classifying as undergraduate or graduate was considered enough to aid in simplifying the search. In some cases courses were used for both undergraduate and graduate programs and this is represented in the table. As an example, 
there were twenty eight courses that had identified topics at the undergraduate only level.

5.1.3 Course Categories. The courses analyzed were categorized through the assignment of a theme. A theme identifies the main focus of a specific course and is a useful approach to categorization since searching a repository based on an overall course theme is something many educators will be comfortable with. The concept of a theme is also simple and easy to understand and is in alignment with the knowledge areas currently of importance in the computing industry, such as cybersecurity and data analytics as covered in the 2018 WG report [13]. A total of seven themes were found to succinctly summarise the entire spectrum of cloud computing courses that were analyzed. Subsequently, when the concept of categorization based on each of the course themes evolved, additional manual searches were conducted to identify courses that were aligned with such themes. As a result, the analysis enabled the discovery of themes that are still emerging and likely to grow in importance in the coming years.

The list shown below presents each of the themes discovered, along with a detailed description of the characteristics of each theme:

- Data-focused Concerned with the migration of data to the cloud. In some cases, this involves lift and shift, where an existing traditional database server containing the data is virtualized and hosted in the cloud. It can also involve porting of existing data of this type to cloud-hosted storage such as NoSQL tables or blob storage. In addition, it can involve using cloud storage technology for the storage of newly gathered data generated by a cloud-hosted application or externally connected system or device. It can also be focused on traditional extract, transform and load (ETL) procedures used in the preparation, manipulation, and querying of data, implemented using available cloud services.

- Application-focused Concerned with the migration of applications and their associated persistence layer(s) to the cloud. In some cases, this involves lift and shift where an existing application deployed to locally hosted servers is deployed to a cloud hosted version of those servers. It can also involve the porting of existing application logic to the cloud using application-specific cloud services technology provided by cloud vendors. In addition, it can involve the creation of completely new applications in the cloud using these vendor-specific technologies.

- Process-focused Concerned with using cloud technology to support the software engineering process. Typically the cloud-based services used are version control, continuous integration / continuous deployment (CI/CD) and artefact repositories. Also included is detailed coverage of process approaches such as Scrum, XP and Agile. Courses with this theme are often concerned with DevOps and use vendorspecific cloud-hosted DevOps services.

- Analytics/ML-focused Concerned with the use of cloud services to support data analytics and machine learning using various interactive and batch analytics cloud frameworks. Vendor-specific offerings are often covered in detail. Also

involves coverage of artificial intelligence theoretical underpinnings.

- Cloud-native-focused Involves the adoption of cloudnative design patterns. Concerned with cloud-hosted services that support DevOps as covered in the process-focused theme as well as employing the twelve-factor app methodology in the construction of applications. In addition, cloudnative employs cloud PaaS services, multi-cloud, microservices and container orchestration services such as Kubernetes as well as instrumentation/monitoring and diagnostic services.

- Security-focused Concerned with the security challenges and mitigation methods for cloud-based deployments. This covers cybersecurity, cross-cutting security analysis, and practices that are important in securing cloud infrastructure, applications, and data. It also covers traditional security technology as well as its implementation using the cloud-specific technologies and security features provided by cloud vendors.

- Infrastructure-focused Concerned with the coverage of virtualization, the cloud software stack, networking etc. provided by cloud vendors. Typical courses are heavily focused on high performance computing (HPC), internet of things (IoT), and networking.

Table 2: Course Themes mapped to KAs
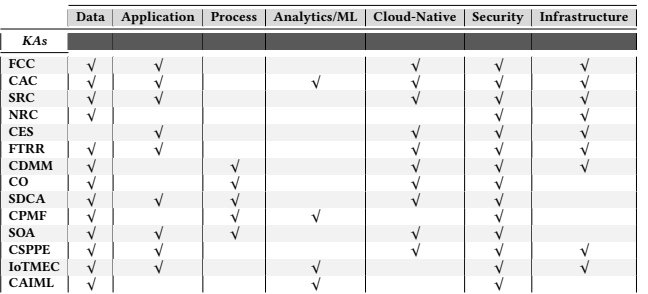

$\mathrm{KA}$ acronyms in the table are listed in Appendix A.

5.1.4 Faculty Willingness to Contribute Materials. The survey included questions on faculty dissemination of their cloud materials and the results of teaching their cloud courses. In response to the question "Would you be willing to share labs/projects/assignments with the community?", 14 faculty replied, "Yes" and 10 replied "Maybe," with just 3 replying "No". Nearly $90 \%$ of respondents indicated they would consider sharing materials and two thirds were interested in sharing their experiences adopting cloud curriculum. In response to "Are you currently researching/publishing your experience with adopting cloud computing in the curriculum?", 11 faculty replied "Yes," 7 replied "Maybe" and 9 replied "No." When asked where their materials resided, there were a variety of schoolspecific sites, but three respondents used GitHub. One specifically asked "I was thinking to create a website with all materials. Are you doing the same?" 
Almost $90 \%$ of responses indicated faculty would consider sharing materials, with two thirds interested in sharing their experiences adopting cloud curriculum. This indicates sufficient interest to support a learning community.

With the course analysis complete, the focus now pivots to an analysis of the current job market to produce insights into how cloud jobs map to the developed KAs, as well as the produced course themes.

\subsection{Job Market Analysis}

5.2.1 Cloud Job Roles and Sub-roles. Over 250 job advertisements were searched and selected for the initial analysis. The six major toplevel job roles identified from the keyword search of cloud and cloud computing on the target recruitment websites $[7,9,17,21,24,40]$ were:

- Cyber \& Information Security;

- Data, Databases \& Data Management;

- Hardware, Networks, \& Infrastructure;

- Digital Business;

- Project \& Product Management;

- Software Design \& Management.

Job title sub-roles were then grouped against one or more of the above listed major job roles. This was carried out to understand how the relatively large and diverse number of job title sub-roles could be grouped into a manageable hierarchy of top-level job roles. The findings of this approach are presented here in the context of UK and USA employment opportunities for cloud computing during the month of July 2019.

The sub-role search terms used were job titles that are prepopulated for selection on Glassdoor, CWJobs, Indeed, and LinkedIn job search services through their respective websites [7, 9, 17, 21, 24, 40]. Tables 4, 5, 6, and 7 in Appendix C show the sub-role job titles mapped to the major job roles. This demonstrates, in the UK and USA at least, that there are many different cloud sub-role job titles under the major 'hardware, networks, \& infrastructure' and 'software design \& management' job roles.

5.2.2 Mapping Major Job Roles to KAs and Course Themes. Six major job roles were identified in the job role analysis part of this work. These were then mapped to the validated KAs from a previous working group, as well as the course themes produced in the course analysis task of this work.

Table ?? presents the mapping of the job roles to both the course theme categories and the KAs. Each major job role corresponds with an appropriately selected theme. This is with the exception of the Cyber \& Information Security role which sits across all of the course themes, the rationale being security is essential across all environments and themes. The table also shows the allocation of the KAs required for each job role. For example, the Fundamental Cloud Concepts (FCC) KA is mandatory for any cloud-related job role. With the KAs mapped to job roles as part of this work, an educator can view which KA is important for their specific curriculum.

\subsection{Learning Community Creation}

This section will discuss the tools and attributes used in the creation of the community repository. Tool selection, enabling communication, and the contribution template used for depositing learning materials in the repository are discussed.

5.3.1 Repository Tool. Informed by the literature search carried out in the background and related work sections in the online repository space, GitHub was selected as the metadata repository tool for learning resources and other related content. This selection was made after comparing to other similar tools, namely Apache Allura [3] and Gitlab [16]. GitHub is a tool with widespread use; as of November 2018, GitHub hosted over 100 million repositories, and has 31 million registered contributors [44]. It is available to academic audiences at no charge. It has well defined processes for making submissions to a repository and making changes to existing elements. It also allows multiple copies of a single work to coexist in the repository on separate branches (so, for example one general assignment could have separate branches for AWS, Azure, and GCP versions). With the availability of GitHub pages [15], users who just wish to consume resources can interact with a website interface.

Each repository on GitHub has a dedicated project page that hosts the source code files, commit history, issues, and other data associated with the project. For example, an educator can upload learning resources to share in the form of a URL with valuable metadata attached to describe and tag the resource. The use of tags will also make it easier for a user to search for content in a specific area. Given the significant scalability of GitHub and its widespread use, it presents a strong rationale for its selection. A shortcoming of GitHub is that there is no way for authors to get direct feedback on how many people viewed or downloaded their materials.

5.3.2 Communication and Support. A wide range of group-based communication tools are available to complement the repository. A search by WG members for a group-based communication tool to support community communication in the repository found the Spectrum.chat [37] tool to be a good fit, with an almost like-for-like Slack [36] feature-set. Slack is one of the most popular group-based communication tools available, with more than 10 million daily users, with academic research exploring its use in organisations [38]. An important benefit of selecting Spectrum.chat is its recent acquisition by GitHub, with a statement made that communities using Spectrum can expect much deeper integration with GitHub [22]. This makes GitHub and Spectrum.chat an ideal combination to support both the technical attributes of the repository and community engagement. The eventual integration of Spectrum.chat features into GitHub can help to improve and enhance community efforts and interactions with other members.

5.3.3 Contribution Template. In order for an educator to make a contribution to the repository, they will use a provided custom template developed by the WG. The template provides mandatory and optional fields to populate, and describes, by use of tags, the uploaded resource. Use of a template facilitates curation of learning resources that can be optimally searched and validated.

The developed template contains the fields below:

- Title

- Summary 
- Author or Contributor (optional)

- Including name, contact information, and institution/company

- Content Link

- Knowledge Area(s)

- Selected from a list of all KAs

- Material Theme(s)

- Selected from a list of all themes.

- Tags to describe the contribution

- Level of Study

* Select from Introduction, Intermediate, Advanced

- Cloud Vendor(s)

- General Tags

* Topics, classes, and other tags to help describe the content

- Prerequisites (optional)

- Learning Objectives (optional)

- License (optional)

When a learning resource is uploaded using the template, it is important that the moderation process for the resource be as transparent as possible and made available on the repository. GitHub has a well-established and well-known process for moderation [14] The members of the WG will serve as moderators of the repository at first. As it grows, they will consider alternatives if this proves to be labour intensive. This approach ensures only high-quality materials will be made available to the community.

\section{DISCUSSION}

Cloud computing technologies are evolving at a significant pace and as such can be seen as disruptive, with many challenges and opportunities yet to be exploited. Cloud supports many technology areas. For example, advancements in network bandwidth and latency may have an effect on how applications are deployed on cloud and edge resources. Given the frequency of change and range of cloud technologies, it is necessary to adopt an iterative cycle in the design of curricula, starting with the innovations by academia, cloud service providers, and adoption by industry. Industry adoption examples can then be used in curricula to demonstrate the practical application of cloud technologies. This makes it crucial for a community-driven repository to support educators in their efforts to adopt cloud through uptake of the available learning resources, as well as lowering the barriers to making a contribution.

In order to keep the repository current with up to date materials, the identified course themes, job roles, and KAs must be periodically reviewed to incorporate any new innovations. KAs and course themes must be updated regularly to ensure the latest industry trends and technologies are well represented in the cloud curriculum. One way that the process can be accelerated is through regular evaluations by cloud educators and through interactions with major cloud service providers and industry to identify important and emergent areas. Based on the cloud computing KAs that were previously identified [13], our analysis in this work identified 'cloud managed services' as a potential new KA that should be monitored for possible future inclusion. It is difficult to foresee the upcoming cloud computing trends beyond the near future due to the rapid rate of change. Therefore, we propose providing updates to the KAs and themes every two years, with the first update to come in 2020 , two years after the first WG report.

\subsection{Importance of Mapping Course Themes, Job Roles, and KAs}

Fundamentally, the existing course analysis mapping task enables educators to search and view learning resources in the repository, including searching against course theme, job role, and KA metadata by using tags. With the tags embedded in the community repository, educators can make an informed decision on the cloud knowledge and skills that are a good fit for their curricula, as well as student career orientation in terms of cloud job roles.

Developing and using custom tags is not a new concept. Instead it builds on the foundation of numerous classification systems, most notably the 2012 ACM Computing Classification System (CCS) [1], which is well established in the computing faculty community. And just as the CCS undergoes periodic updating, these mappings will need to be maintained. The CCS only contains four references to cloud computing, so the mapping done as part of this work is much more detailed about cloud concepts.

6.1.1 Course Themes. A goal of the existing course analysis task was to develop meaningful categorization of course themes. The developed categories were designed to be presented to educators as accessible and clearly defined cloud topics. The derived categories are used to tag course content in the repository. The course tags are primarily presented on the repository for educators to search for content, as well as mandating their selection when making a course contribution. Categories that are too detailed will lead to course identification becoming conflated, while too few categories will lead to educators spending a lot of time filtering the course list when attempting to search for appropriate content. Overall, the goal was to reach a categorization approach that was not skewed to being either abstract or too detailed. It is expected that as the field of cloud computing evolves and different courses are developed, so too will the tags used in the repository. The repository will facilitate the dynamic updating, addition, and removal of tags as necessary. However, to encourage adoption from the offset, a predefined set of tags will be available on the repository as developed from the existing course analysis data.

6.1.2 Job Roles. The job role analysis produced 6 primary job role areas as shown previously in Table ??. The job roles were then mapped over to a single course theme and appropriate KAs to produce an effective grouping mechanism for cloud learning resources. For example, the job role 'hardware, network, \& infrastructure' is allocated to the 'infrastructure' course theme, as well as the following KAs: FCC, CAC, NRC, CES, FTRR, CDMM, CO, and IoTMEC. Within this job role, a typical job title would be 'cloud infrastructure engineer', which has the job requirement as follows: "Ability to configure, monitor and troubleshooting of network infrastructure devices, cloud-based network security groups (NSG), Wi-Fi access points, routers, switches, servers (physical and virtual), UPSs, network and application load balancers, VPN and remote access solutions, remote access services support, access control, and identity, management task" [20]. 
From the job title and description analysis, an informed selection of 'best-fit' KAs was facilitated. This approach also helps to validate the KAs in terms of their relevance, in that all the KAs were found to be represented in the searched job advertisements, as well as the identification of potential new KAs. Of note the 'cyber \& information security' job role requires all KAs due to its importance and consideration in all aspects of cloud computing, and the KAs are well represented across the spectrum of cloud jobs as of July 2019.

\subsection{Learning Community Considerations}

As previously discussed in the Related Work section, there are attributes of a teaching repository that are considered critical for its success in terms of sustaining engagement. This work investigated the use of suitable tools to support the main technical components of a repository as well as that of the required community of endusers. A single tool that supports both the technical and end-user interactions and communication attributes could not be found online after an extensive search. The examples of teaching repositories previously discussed were custom built software with limited features, this also introduces the overhead of hosting and general maintenance. Additionally, none of the teaching repositories identified supported any rich communication features such as those commonly found in contemporary messaging tools, particularly group features and file sharing. Without a rich communication set, the community would be constrained in its efforts for tightly coupled collaboration and sharing.

In addition to the repository design being informed by previous academic literature, the results of the course and job analysis tasks directly informed the repository feature-set. One such key feature is the capability of tagging, which is described next in more detail.

6.2.1 Tagging. With the primary purpose of the repository to act as an enabler of collaboration, uptake, and sharing of teaching materials, consideration to the roles of an educator as both a consumer and contributor need to be understood and facilitated. In the consumer role scenario, the educator will likely have a few predefined search terms they would like to use that are linked to cloud computing, e.g., virtualisation, PaaS, IaaS, DevOps, etc. They also may prefer to filter their searches by difficulty level, such as beginner, intermediate, and advanced. For example an educator new to cloud computing may wish to search for courses suitable for beginners, such as the use of PaaS services. The repository can provide bespoke searching and filtering through the use of custom tags (GitHub feature) that can be utilised, with tags applied to uploaded learning resources. In summary, the role of consuming (via search) learning resources from the repository is well provided for by the tagging and moderation of uploaded materials.

\section{SUMMARY AND FUTURE WORK}

Given the current industry skills-gap in cloud computing, complexity of the area, and the rapid pace of industry innovation, the question of how educators in higher education can incorporate cloud courses into their curricula is a timely one and the focus of the work presented in this paper. Findings from a set of tasks that worked toward the creation of a community-driven learning repository for cloud computing learning resources were presented.
The repository was designed for educators to search for, and contribute, high-quality cloud learning materials. We believe that such a community-driven and contextualized to industry collection of learning materials will enable a collaborative effort around the production and sharing of cloud curricula materials, enabling timely adoption of cloud in higher education institutions.

Our work in this paper is a continuation of the work carried out by the ITiCSE 2018 WG [9], in which the authors set about addressing the significant challenge of exploring the landscape and state of cloud computing in higher education. The 2018 WG resulted in the production of fourteen identified knowledge areas in the domain of cloud computing, it also revealed there was very little in the way of cloud learning resources available to educators.

The lack of learning materials was the primary motivation for this work and prompted the development of the three objectives. The first was to extend the work done by the 2018 WG by thematically analyzing cloud computing courses currently taught in higher education, and mapping the developed course themes to the original knowledge areas. This approach allowed the WG to gauge how pervasive the knowledge areas are represented in current cloud curricula. The second was to conduct a search of current cloud job postings to produce a list of cloud job roles that the majority of job titles could fit under; these job roles were then mapped to both the course themes and knowledge areas. Finally, the course theme and job role findings were used in the creation of a community-driven learning repository, where educators can search for, and contribute, cloud learning materials.

Our planned future work includes:

- Monitor our curriculum repository and make improvements for quality and curation purposes;

- Monitor the community needs and make adjustments as necessary;

- Disseminate information about the availability of the community and curriculum repository to the larger computer science education community, and ask for participation;

- Interact with curriculum recommendations [2] and explore how to develop further;

- Monitor the state of cloud computing education and update KAs, LOs, themes, and tags as appropriate.

\section{A THE KNOWLEDGE AREAS}

The proposed Knowledge Areas from [13] are:

- Fundamental Cloud Concepts (FCC)

- Computing Abstractions on the Cloud (CAC)

- Storage Resources on the Cloud (SRC)

- Networking Resources on the Cloud (NRC)

- Cloud Elasticity and Scalability (CES)

- Fault Tolerance, Resilience and Reliability (FTRR)

- Cloud Deployment, Monitoring and Maintenance (CDMM)

- Cloud Orchestration (CO)

- Software Development using Cloud APIs (SDCA)

- Cloud Programming Models and Frameworks (CPMF)

- Service Oriented Architecture (SOA)

- Cloud Security, Privacy, Policy and Ethics (CSPPE)

- IoT, Mobile, Edge and the Cloud (IoTMEC) 
- Cloud-based Artificial Intelligence and Machine Learning (CAIML)

\section{B FACULTY SURVEY}

\section{B.1 Academic Cloud Courses Survey - ACM ITiCSE 2019 Working Group}

A working group titled "Toward Developing a Cloud Computing Model Curriculum" is exploring the current state of adoption of cloud computing across different computing courses and programs at a broad range of higher education institutions. The working group will convene at the 24th Annual Conference on Innovation and Technology in Computer Science Education (ITiCSE 2019) (https://iticse.acm.org/). More information about the working group: WG7 at https://iticse.acm.org/working-group-details/\#WG7.

Please submit one survey per course. Click next to Section 2 to enter your information. Then, click next to Section 3 to enter information about the course. The survey should take about 5-10 minutes to complete. Thank you!

B.1.1 Faculty and Institution Information. Please enter your and institution information below and then click next. Thank you!

- Name:

- Email:

- Personal Web Page URL:

- Field/Area of Focus (Cloud Computing, Data Science, Cybersecurity, Machine Learning, AI, etc.):

- Job Title:

- Department:

- Institution Name:

- Country:

B.1.2 Course Information. Please enter your course information below and then click submit. Thank you!

- Do you teach more than one cloud-related course?

- Yes (Please complete one survey for each course)

- No

- Target audience

- Associate

- Bachelor's

- Master's

- Doctorate/PhD

- Non-degree certificate

- Professional development

- Other:

- Course Title:

- Course Code:

- Course Description:

- URL to Course Syllabus:

- Course Learning Objectives:

- Course Web Page:

- How many times have you offered the course?

$-1$

$-2$

$-3$

$-4$

- Other:
- Date you first taught the cloud-related course:

- Average number of students in the course per offering:

- What is the delivery method?

- Face-to-face

- Blended/Hybrid

- Online

- Other:

- Please list the major topic areas in your course (Virtualization, Microservices, Storage, Analytics, Edge, etc.):

- Number of individual written assignments

- None

$-1-5$

$-5-10$

$-10+$

- Number of INDIVIDUAL hands-on labs/projects

- None

$-1-5$

$-5-10$

$-10+$

- Number of TEAM hands-on labs/projects

- None

$-1$

$-2$

$-3$

- Other:

- Number of assessments/quizzes/exams

- None

$-1$

$-2$

$-3$

- Other:

- The percentage of the course grade from all individual written assignments

- 11-point scale from $0 \%$ to $100 \%$

- The percentage of the course grade from all INDIVIDUAL hands-on labs/projects

- 11-point scale from $0 \%$ to $100 \%$

- The percentage of the course grade from all TEAM hands-on labs/projects

- 11-point scale from $0 \%$ to $100 \%$

- The percentage of the course grade from all assessments/quizzes/exams

- 11-point scale from $0 \%$ to $100 \%$

- Which cloud technologies have you used in part of the course?

- AWS

- Microsoft Azure

- Google Cloud

- IBM

- On-Premise OpenStack

- On-Premise VMWare

- None

- Other:

- Who pays for the cloud usage

- Institution

- Course Grant/Sponsor (Cloud Credits)

- Student Offer (Cloud Credits) 
- Other:

- Please list any cloud services used (AWS EMR, Azure HDInsight, GCP Dataproc, etc.):

- Please list any tools used (Jupyter, R, Tableau, etc.):

- Share more about your hands-on projects/labs/assignments (topics/project descriptions/GitHub Repos/Content):

- Would you be willing to share labs/projects/assignments with the community?

- Yes

- No

- Maybe
- Are you currently researching/publishing your experience with adopting cloud computing in the curriculum?

- Yes

- No

- Maybe

- Please share links to any existing materials/publications:

\section{DETAILED JOB POSTING INFORMATION}

Tables 4 through 7 in this section give details for the jobs that were evaluated as part of analyzing the job market.

Table 4: Cloud Job Role - H/W, Networks, \& Infrastructure

\begin{tabular}{|c|c|c|c|c|c|c|c|}
\hline \multirow[b]{2}{*}{ Sub-role Title } & \multicolumn{4}{|c|}{ UK } & \multicolumn{3}{|c|}{ USA } \\
\hline & Glassdoor & CW Jobs & indeed & LinkedIn & Glassdoor & indeed & LinkedIn \\
\hline Cloud Solution Architect & 147 & 82 & 93 & 366 & 262 & 530 & 1,883 \\
\hline Cloud Computing Architect & 98 & 89 & 40 & 9 & 2 & 318 & 140 \\
\hline AWS Cloud Software Architect & 58 & 20 & 35 & 18 & 2 & 496 & 285 \\
\hline Cloud Infrastructure Architect & 173 & 94 & 112 & 168 & 63 & 131 & 374 \\
\hline Cloud Architect & 173 & 94 & 112 & 151 & 779 & 1,131 & 1,238 \\
\hline Cloud Application Architect & 102 & 48 & 424 & 8 & 26 & 4,604 & 249 \\
\hline Cloud Native Architect & 5 & 6 & 42 & 17 & 5 & 235 & 224 \\
\hline Cloud Native Computing and DevOps Solution Architect & 2 & - & 6 & 6 & 40 & 41 & 40 \\
\hline Cloud Orchestration Architect & 12 & 4 & 21 & 32 & 798 & 379 & 294 \\
\hline Cloud Network Architect & 173 & 52 & 147 & 158 & 6 & 2,974 & 727 \\
\hline Network Engineer, Cloud Computing Networks & 222 & 24 & 120 & 24 & 2,737 & 491 & 332 \\
\hline Cloud Engineer & 444 & 392 & 418 & 383 & 1,282 & 7,562 & 1,660 \\
\hline Cloud Computing Engineer & 733 & 372 & 120 & 24 & 20 & 1,752 & 176 \\
\hline Cloud Senior Engineer & 655 & 99 & 149 & 93 & 132 & 2,609 & 888 \\
\hline Cloud Engineering Specialist & 52 & 260 & 42 & 21 & 2 & 478 & 70 \\
\hline Cloud Engineer Computing & 222 & 257 & 120 & 70 & 15 & 1,752 & 1,050 \\
\hline Cloud Systems Engineer & 387 & 215 & 356 & 137 & 118 & 6,376 & 1,892 \\
\hline Cloud Infrastructure Engineer & 444 & 392 & 418 & 157 & 125 & 7,562 & 696 \\
\hline
\end{tabular}

Table 5: Cloud Job Role - Project \& Product Management

\begin{tabular}{|c|c|c|c|c|c|c|c|}
\hline \multirow[b]{2}{*}{ Sub-role Title } & \multicolumn{4}{|c|}{ UK } & \multicolumn{3}{|c|}{ USA } \\
\hline & Glassdoor & CW Jobs & indeed & LinkedIn & Glassdoor & indeed & LinkedIn \\
\hline Cloud Solution Architect & 720 & 26 & 867 & 6 & 262 & 1,743 & 122 \\
\hline Cloud Native Computing and DevOps Solution Architect & 17 & - & 4 & - & 40 & 21 & 6 \\
\hline Cloud Orchestration Architect & 67 & 2 & 50 & 4 & 66 & 149 & 34 \\
\hline Cloud Administrator & 393 & 2 & 153 & 1 & 68 & 488 & 14 \\
\hline Cloud Environment Senior Systems Administrator & 90 & 4 & 26 & - & 1 & 94 & 7 \\
\hline Cloud Computing DevOps Engineer & 105 & 2 & 62 & 1 & 1 & 197 & 7 \\
\hline Cloud Automation Engineer & 355 & 22 & 411 & - & 1 & 1,242 & 19 \\
\hline Cloud DevOps and IT Operations & 166 & - & 180 & 6 & 2,383 & 774 & 57 \\
\hline Cloud DevOps and Infrastructure Engineer & 166 & 6 & 236 & 6 & 3,857 & 632 & 8 \\
\hline
\end{tabular}


Table 6: Cloud Job Role - Data, Databases, \& Data Management

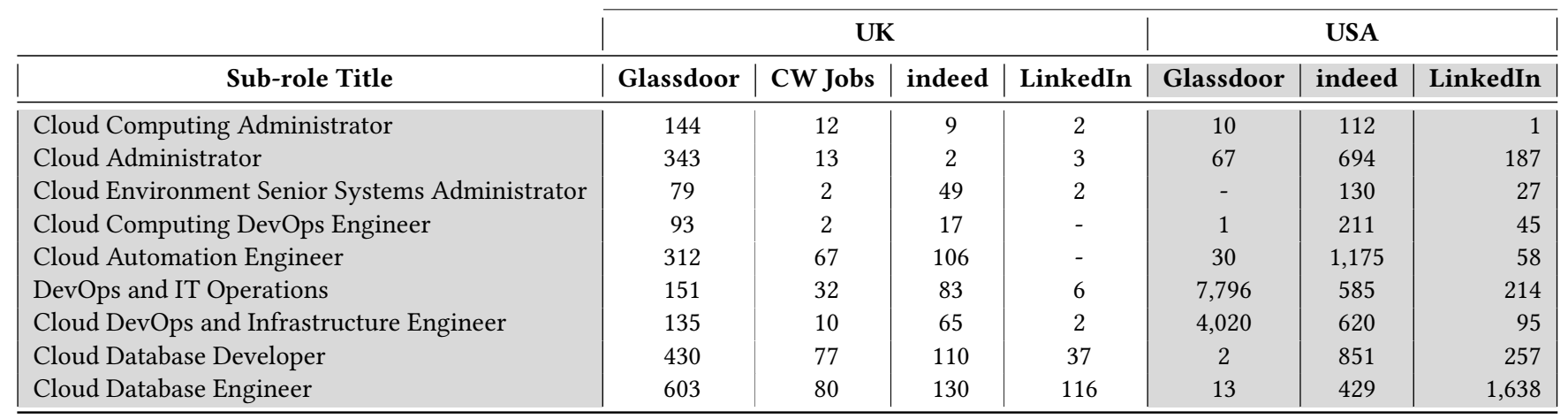

Table 7: Cloud Job Role - Cyber \& Information Security

\begin{tabular}{|l|r|r|r|r|r|r|r|}
\cline { 2 - 7 } & \multicolumn{4}{c}{ UK } & \multicolumn{3}{c|}{ USA } \\
\hline \multicolumn{1}{c|}{ Sub-role Title } & Glassdoor & CW Jobs & indeed & LinkedIn & Glassdoor & indeed & LinkedIn \\
\hline Cloud Security Architect & 148 & 46 & 767 & 523 & 125 & 2,914 & 1,557 \\
Cloud Security Manager & 195 & 45 & 987 & 861 & 1 & 3,662 & 994 \\
Cloud Engineer - Security System & 120 & 5 & 912 & 4 & 2,330 & 7,363 & 41 \\
Cloud Security Assessor & 2 & 261 & 19 & 4 & 3 & 132 & 247 \\
Cloud Security Engineer & 144 & 164 & 1,241 & 498 & 189 & 121,926 & 1,723 \\
Cloud Security Developer & 30 & 64 & 532 & 649 & 20 & 4,306 & 1,454 \\
Cloud Security Analyst & 148 & 39 & 395 & 141 & 2 & 3,038 & 882 \\
Cloud Penetration Tester & 10 & 9 & 22 & 28 & 1 & 97 & 150 \\
Cloud Security Infrastructure & 82 & 307 & 721 & 334 & 4 & 7,175 & 680 \\
Principal Cloud Security Architect & 110 & 12 & 38 & 9 & 63 & 449 & 145 \\
\hline
\end{tabular}

\section{REFERENCES}

[1] ACM Inc. 2019. The 2012 ACM Computing Classification System. https: //www.acm.org/publications/class-2012. Retrieved 14 September 2019.

[2] ACM Inc. 2019. Curricula Recommendations. https://www.acm.org/education/ curricula-recommendations. Retrieved 14 September 2019.

[3] Apache Foundation. 2019. Apache Allura. https://allura.apache.org/.

[4] Jeremy Benson. 2015. Explaining the RQF. https://scqf.org.uk/. Retrieved 12 September 2019.

[5] Blackboard Inc. 2019. Blackboard Education Technology \& Services. https: //uk.blackboard.com/index.html. Retrieved 3 September 2019.

[6] Richard Brown, Libby Shoop, and Joel Adams. 2016. CSinParallel: Parallel Computing in the Computer Science Curriculum. https://csinparallel.org.

[7] CareerBuilder LLC. 2019. Find a Job | CareerBuilder. https://www.careerbuilder com/. Retrieved 14 September 2019.

[8] Richard Cooper and Sally Fincher. 2019. dbdc: Creating a Disciplinary Commons in Computing Education. https://www.cs.kent.ac.uk/people/staff/saf/dbdc/index. html. Retrieved 12 September 2019.

[9] Dice. 2019. Find Jobs in Tech | Dice.com. https://www.dice.com/. Retrieved 14 September 2019.

[10] ECORYS UK. 2016. Digital Skills for the UK Economy. Technical Report. Department for Business Innovation \& Skills.

[11] Education and Culture DG. 2008. The European Qualifications Framework for Lifelong Learning (EQF). Technical Report. European Commission. http: //ecompetences.eu/wp-content/uploads/2013/11/EQF_broch_2008_en.pdf.

[12] Sally Fincher, Michael Kölling, Ian Utting, Neil Brown, and Phil Stevens. 2010 Repositories of Teaching Material and Communities of Use: Nifty Assignments and the Greenroom. In Proceedings of the Sixth International Workshop on Computing Education Research (ICER '10). ACM, New York, NY, USA, 107-114. https://doi.org/10.1145/1839594.1839613
[13] Derek Foster, Laurie White, Joshua Adams, D. Cenk Erdil, Harvey Hyman, Stan Kurkovsky, Majd Sakr, and Lee Stott. 2018. Cloud computing: developing contemporary computer science curriculum for a cloud-first future. In Proceedings Companion of the 23rd Annual ACM Conference on Innovation and Technology in Computer Science Education, ITiCSE 2018, Larnaca, Cyprus, fuly 02-04, 2018. 130-147. https://doi.org/10.1145/3293881.3295781

[14] GitHub Inc. 2019. About pull requests - GitHub Help. https://help.github.com/ en/articles/about-pull-request-reviews. Retrieved 14 September 2019.

[15] GitHub Inc. 2019. GitHub Pages. https://pages.github.com/. Retrieved 12 September 2019.

[16] Gitlab. 2019. The first single application for the entire DevOps cycle. https: //about.gitlab.com/.

[17] Glassdoor Inc. 2019. Glassdoor Job Search. https://www.glassdoor.com/index.htm. Retrieved 14 September 2019.

[18] Susan Imberman and Ann Fiddler. 2019. Share and Share Alike:: Using Creative Commons Licenses to Create OER. ACM Inroads 10, 2 (April 2019), 16-21. https://doi.org/10.1145/3324887

[19] Instructure Inc. 2019. Instructure. https://instructure.com. Retrieved 4 September 2019.

[20] Indeed. 2019. Cloud Platform \& Infrastructure Engineer. https://www.indeed. com/viewjob?jk=0b247eb496b7ec19\&from=myjobs\&tk=1dij760rch2pc800. Retrieved 14 September 2019.

[21] Indeed. 2019. Job Search | Indeed. https://www.indeed.com/. Retrieved 14 September 2019.

[22] Bryn Jackson. 2018. Spectrum is joining GitHub! https://spectrum.chat/spectrum/ general/spectrum-is-joining-github 1d3eb8ee-4c99-46c0-8daf-ca35a96be6ce. Retrieved 14 September 2019.

[23] William A Kahn. 1990. Psychological conditions of personal engagement and disengagement at work. Academy of management journal 33, 4 (1990), 692-724.

[24] LinkedIn Corporation. 2019. LinkedIn. https://www.linkedin.com/. Retrieved 14 September 2019. 
[25] Microsoft. 2019. Meet Microsoft Teams - Microsoft in Education. https://education. microsoft.com/courses-and-resources/resources/meet-microsoft-teams. Retrieved 4 September 2019.

[26] NNhlanhla Mlitwa. 2006. s (LMS) in a changing higher education environment. In Transforming IS \& CS Education and Research in a Changing Higher Education Environment. E-Innovation Academy, Cape Peninsula University of Technology.

[27] Moodle Pty Ltd. 2019. Moodle - Open-source learning platform. https://moodle org. Retrieved 4 September 2019.

[28] Lijun Ni, Mark Guzdial, Allison Elliott Tew, Briana Morrison, and Ria Galanos 2011. Building a Community to Support HS CS Teachers: The Disciplinary Commons for Computing Educators. In Proceedings of the 42Nd ACM Technical Symposium on Computer Science Education (SIGCSE '11). ACM, New York, NY, USA, 553-558. https://doi.org/10.1145/1953163.1953319

[29] Sushil K. Prasad, Almadena Chtchelkanova, Frank Dehne, Mohamed Gouda, Joseph Jaja, Krishna Kant, Anita La Salle, Richard LeBlanc, Andrew Lumsdaine, David Padua, Manish Parashar, Viktor Prasanna, Yves Robert, Arnold Rosenberg, Sartaj Sahni, Behrooz Shirazi, Alan Sussman, Chip Weems, and Jie Wu. 2012. NSF/IEEE-TCPP Curriculum Initiative on Parallel and Distributed Computing - Core Topics for Undergraduates, Version I. http://www.cs.gsu.edu/ tcpp/ curriculum.

[30] Soumya Ray, Sung S. Kim, and James G. Morris. 2014. The Central Role of Engagement in Online Communities. Info. Sys. Research 25, 3 (9 2014), 528-546.

[31] Russell Group of Universities. 2019. Russell Group. https://russellgroup.ac.uk/. Retrieved 9 September 2019.

[32] Renu Sabharwal, Rahat Hossain, Ritesh Chugh, and Marilyn Wells. 2018. Learning Management Systems in the Workplace: A Literature Review. In 2018 IEEE International Conference on Teaching, Assessment, and Learning for Engineering (TALE). IEEE, 387-393. https://doi.org/10.1109/TALE.2018.8615158

[33] Rainer Schmidt, Michael Möhring, Ralf-Christian Härting, Christopher Reichstein, Pascal Neumaier, and Philip Jozinovic. 2015. Industry 4.0 - Potentials for
Creating Smart Products: Empirical Research Results. In BIS.

[34] Scottish Credit and Qualifications Framework Partnership. 2019. SCQF | Scottish Credit and Qualifications Framework. https://scqf.org.uk/. Retrieved 12 September 2019.

[35] Sue Sentance and Andrew Csizmadia. 2017. Professional Recognition Matters: Certification for In-service Computer Science Teachers. In Proceedings of the 2017 ACM SIGCSE Technical Symposium on Computer Science Education (SIGCSE '17). ACM, New York, NY, USA, 537-542. https://doi.org/10.1145/3017680.3017752

[36] Slack. 2019. Where work happens | Slack. https://slack.com/. Retrieved 14 September 2019.

[37] Spectrum. 2019. Spectrum. https://spectrum.chat/. Retrieved 14 September 2019.

[38] Viktoria Stray, Nils Brede Moe, and Mehdi Noroozi. 2019. Slack Me if You Can!: Using Enterprise Social Networking Tools in Virtual Agile Teams. In Proceedings of the 14th International Conference on Global Software Engineering (ICGSE '19). IEEE Press, Piscataway, NJ, USA, 101-111. https://doi.org/10.1109/ICGSE.2019.00031

[39] Josh Tenenberg and Sally Fincher. 2007. Opening the Door of the Computer Science Classroom: The Disciplinary Commons. SIGCSE Bull. 39, 1 (March 2007), 514-518. https://doi.org/10.1145/1227504.1227484

[40] Totaljobs Group Ltd. 2019. The UK IT Jobs Board at CWJobs.co.uk. https: //www.cwjobs.co.uk/. Retrieved 14 September 2019.

[41] Argiris Tzikopoulos, Nikos Manouselis, and Riina Vuorikari. 2007. An overview of learning object repositories. In Learning objects for instruction: Design and evaluation, Pamela T. Northrup (Ed.). Information Science Publishing, 29-55.

[42] UCAS. 2019. UCAS | At the heart of connecting people to higher education. https://www.ucas.com/. Retrieved 4 September 2019.

[43] University Alliance Limited. 2019. University Alliance. https://www.unialliance. ac.uk/. Retrieved 9 September 2019.

[44] Jason Warner. 2018. Thank you for 100 million repositories. https://github.blog/ 2018-11-08-100m-repos/. Retrieved 12 September 2019. 